\title{
Histone deacetylase inhibition reduces deleterious cytokine release induced by ingenol stimulation
}

Erin T. Larragoite ${ }^{* 1}$, Laura J. Martins ${ }^{* 1}$, Adam M. Spivak ${ }^{* 2}$, Racheal A. Nell ${ }^{2}$, Vicente Planelles ${ }^{1 \S}$ ${ }^{*}$ Contributed equally

Authors' Affiliation: ${ }^{1}$ Department of Pathology, University of Utah, Salt Lake City, United States;

${ }^{2}$ Department of Medicine, University of Utah School of Medicine, Salt Lake City, United States

${ }^{\S}$ Corresponding Author: Vicente Planelles, vicente.planelles@path.utah.edu

ETL erin.larragoite@path.utah.edu

LM laura.martins@path.utah.edu

AMS adam.spivak@hsc.utah.edu

RN Racheal.Nell@hsc.utah.edu

VP vicente.planelles@path.utah.edu

\begin{abstract}
Introduction: Though antiretroviral therapy has led to viral suppression and increased quality of life for patients living with HIV-1, strategies to eliminate the HIV-1 latent reservoir are still necessary to eliminate HIV. Latency reversal with superior latency reversal agents (LRAs) such as protein kinase $\mathrm{C}$ (PKC) agonists is a promising strategy for unveiling and eliminating the latent HIV-1 reservoir. However, PKC agonists induce T cell activation and deleterious proinflammatory cytokine production. Secondary pharmacological agents combined with LRAs have been previously shown to reduce deleterious pro-inflammatory cytokine secretion without inhibiting HIV-1 viral reactivation. Histone deacetylase inhibitors (HDACi) are also known for inhibiting deleterious pro-inflammatory cytokines in the context of graft-versus-host disease and rheumatoid arthritis in addition to being known to synergize with PKC agonists. In this study we investigated whether HDACi and other epigenetic modifiers could decrease PKCinduced pro-inflammatory cytokines secretion while simultaneously synergizing with the PKC agonists Ingenol-3,20-dibenzoate, to enhance latency reversal.

Methods: We screened an epigenetic modifier library in health donor human peripheral blood mononuclear cells (PBMCs) to identify compounds ('hits') that reduced intracellular IL-6 pro-inflammatory cytokine production induced by PKC agonist Ingenol-3,20-dibenzoate. We
\end{abstract}


then further tested reducers of intracellular IL-6 ('hits') for their ability to synergize with Ingenol-3,20-dibenzoate in the J-LAT 10.6 model of HIV-1 latency. The most promising epigenetic modifier from both screens, the HDACi Panobinostat, was then further tested for its ability to reduce pro-inflammatory cytokines and synergize with Ingenol-3,20-dibenzoate.

Results: We show that co-treatment with Ingenol-3,20-dibenzoate and Panobinostat reduces pro-inflammatory cytokines and enhances latency reversal in vitro. Panobinostat suppressed pro-inflammatory cytokine production when combined with Ingenol-3,20dibenzoate ex vivo when using aviremic patient cells, but antagonized Ingenol-3,20-dibenzoate dependent latency reversal ex vivo.

Conclusion: The combination of Panobinostat and Ingenol-3,20-dibenzoate reduces deleterious cytokine production but is not a suitable latency reversal combination therapy.

Keywords: HIV-1; Latency Reversing Agent; Ingenol-3,20-dibenzoate; Panobinostat; Synergy; Antagonist; Cytokine

\section{Introduction}

Antiretroviral therapy (ART) has profoundly improved the lives of individuals living with HIV-1 by reducing plasma viremia levels below the limit of detection ( 20-50 copies/ml) [1-3] and allowing for reconstitution of the adaptive immune system. However, ART is unable to eradicate HIV-1 due to the presence of a silent, but long-lived latent viral reservoir [4-6]. The presence of this latent reservoir prevents patients from being removed from ART, making ART a lifelong necessity [7]. Additionally, not all patients have access to ART or are compliant with ART regiments due to high costs [8], side effects [9], and social stigmas [10, 11]. Aviremic individuals taking ART are prone to immune dysfunction conditions [12] and neurologic complications associated with HIV-1 [13] underscoring the importance of strategies targeting the latent HIV-1 reservoir.

Many strategies have been proposed to eliminate the latent HIV-1 reservoir including the "shock and kill" strategy [14], in which latency reversing agents (LRAs) are used to transcriptionally reactivate the latent reservoir prior to targeting with immune-mediated 
mechanisms $[14,15]$. Additional strategies include the use of broadly neutralizing antibodies (bNAbs), cytokines, vaccines or other immune-mediated mechanisms to enhance recognition of HIV-1 infected cells via the immune system $[16,17]$ and identification of cellular markers specific to cells harboring latent HIV-1 to target latently infected cells [12]. While all strategies hold merit, enhanced targeting of latently infected cells via immune-mediated mechanisms would still be dependent on unveiling the latent reservoir. Therefore, unveiling latent HIV-1 via the use of LRAs may be necessary before additional targeting of infected cells.

Several classes of compounds have emerged as potential LRAs including positive transcription elongation factor b (PTEFb) activators, cytokines, histone deacetylase inhibitors (HDACi), and protein kinase C (PKC) agonists $[15,18]$. However, not all LRAs appear to be created equal. Spina et al. [18] demonstrated that PKC agonists are superior LRAs, shown to reactivate latent HIV-1 across multiple models of HIV-1 latency, whereas other well characterized LRAs such as HDACi were unable to reactivate to the same extent in some models of HIV-1 latency. Additionally, PKC agonists have been shown to reactivate latent provirus and lead to cell death of HIV-1 infected cells [19]. PKC agonists are thought to reactivate latent HIV1 though activation of NF-KB and have been shown to additionally activate AP-1 and NFAT [20]. While PKC agonists are superior LRAs, they also induce T cell activation [21] and proinflammatory cytokine secretion. Spivak et al. [22] first demonstrated that latency reversal with PKC agonist Ingenol-3,20-dibenzoate used in combination with a second pharmacological agent, Ruxolitinib, an FDA approved Janus Kinase (JAK) inhibitor, to suppress deleterious proinflammatory cytokine secretion, led to potent reactivation of latent HIV-1 and suppression of pro-inflammatory cytokine secretion induced by Ingenol compounds. Additionally, Rapamycin, an inhibitor of mTOR, has been shown to reduce pro-inflammatory cytokine secretion induced upon reactivation of latent HIV-1 with $\alpha \mathrm{CD} 3 / \alpha C D 28$ [23]. These studies suggest that latency reversal and cytokine secretion can be uncoupled and that potent reactivation with PKC agonists may be achieved without deleterious side effects of cytokine release. Therefore, we were interested in determining if additional compounds could be used to inhibit deleterious cytokines upon reactivation of latent HIV-1 with PKC agonists. 
While Ruxolitinib and Rapamycin have been shown to reduce in vivo cytokine secretion in the context of HIV-1, HDACi have also been well characterized to inhibit pro-inflammatory cytokine secretion in vivo as a result of graft-versus-host disease [24] and rheumatoid arthritis [25] as well as in vitro, as a result of LPS stimulation of human peripheral blood mononuclear cells (PBMCs) [26]. Additionally, HDACi are well known LRAs and although they have been characterized as being less potent LRAs than PKC agonists, HDACi have been shown to synergize with PKC agonists when reactivating latent HIV-1 [27-29]. Therefore, we hypothesized that HDACi and other epigenetic modifiers could decrease PKC-induced pro-inflammatory cytokines secretion while simultaneously synergizing with PKC agonists to enhance latency reversal. In this study, we show that Panobinostat, a broad-spectrum HDAC inhibitor, decreases PKCinduced pro-inflammatory cytokines while simultaneously enhancing latency reversal in vitro through synergy with PKC agonist Ingenol-3,20-dibenzoate. While the cytokine suppression ability of Panobinostat was maintained ex vivo when using aviremic patient cells, we observed that Panobinostat failed to synergize with Ingenol-3,20-dibenzoate in this system and, instead, exerted antagonism.

\section{Methods}

\section{Participants}

Healthy donors and aviremic HIV-1 positive patients were recruited in accordance with University of Utah Institutional Review Board (IRB) protocols 67637 (approved May 31, 2017) and 58246 (approved Jan 4, 2017). Aviremic HIV-1 positive participants included in the study were aviremic (plasma viral loads less than 50 HIV-1 RNA copies/mL) for a minimum of 6 months and compliant with an ART regimen initiated during chronic HIV-1 infection for a minimum of 12 months.

\section{In vitro epigenetic inhibitor screening}

PMBCs were isolated from healthy donors using a Lymphoprep density gradient (Cat\# 07861, StemCell Technologies) prior to being cultured in RPMI medium supplemented with $10 \%$ FBS and $5 \mathrm{U} / \mathrm{mL}$ penicillin/streptomycin overnight to remove monocytes via adherence. Non- 
adherent PBMCs were then cultured at a density of $1 \times 10^{5}$ cells $/ 100 \mu \mathrm{L}$ in the presence of 100nM compounds from a Cayman Chemical Epigenetics Screening Library obtained from the University of Utah Drug Discovery Core Facility (Item No. 11076) or 100nM Ruxolitinib, a control for cytokine inhibition, for $1.5 \mathrm{~h}$ at $37^{\circ} \mathrm{C}$. Cells were then incubated in media alone or the presence of $100 \mathrm{nM}$ Ingenol-3,20-dibenzoate for 40 hours post-stimulant exposure. At 40 hours post-stimulant exposure, $0.067 \mu \mathrm{L} / 100 \mu \mathrm{L}$ BD GolgiStop ${ }^{\mathrm{TM}}$ Protein Transport Inhibitor (Cat\# 554724) was added to each sample to inhibit cytokine secretion. Cells were then fixed and stained at 48 hours prior to flow cytometry analysis.

\section{Intracellular cytokine staining and flow cytometry}

Cells were washed with 1x phosphate buffered saline (PBS) prior to staining the cells with $0.1 \mu \mathrm{L} / 100 \mu \mathrm{L}$ Fixable Viability Dye eFluor ${ }^{\circledR} 450$ (Cat\# 65-0863-14, Affymetrix eBioscience) for $30 \mathrm{~min}$ at $4^{\circ} \mathrm{C}$. Cells were then washed with $1 \times$ PBS prior to fixing with $100 \mu \mathrm{LD}$ Cytofix/Cytoperm ${ }^{\mathrm{TM}}$ for $30 \mathrm{~min}$ at $4^{\circ} \mathrm{C}$. Once cells were fixed they were washed with a perm/wash solution (1x PBS, 3\% FBS, 0.1\% Saponin, 0.05\% Sodium Azide) prior to staining cells in $100 \mu \mathrm{L}$ perm/wash with $0.5 \mu \mathrm{L}$ APC anti-human IL-6 antibody (Cat\# 501112, Biolegend ${ }^{\circledR}$ ) overnight at $4^{\circ} \mathrm{C}$. Cells were finally washed in $1 \times$ PBS and re-suspended in PBS prior to flow cytometry [BD FACSCanto $^{\mathrm{TM}}$ flow cytometer with FACSDiva $^{\mathrm{TM}}$ acquisition software (BectonDickinson, Mountain View, CA)] and analysis with FlowJo (TreeStar Inc, Ashland, OR).

\section{Selection of compounds}

The percentage of IL-6-positive cells in ingenol-3,20-dibenzoate-alone-treated cells was compared to cells treated with epigenetic modifiers and Ingenol-3,20-dibenzoate in order to calculate intracellular IL- 6 fold change. Epigenetic modifiers that reduced intracellular IL- 6 by fourfold or greater ('hits') were selected for a secondary screen to examine the ability of these compounds to synergize with Ingenol-3,20-dibenzoate. 


\section{Synergy Screening}

J-LAT 10.6 cells [30] were cultured in RPMI supplemented with $10 \% \mathrm{FBS}$ and $5 \mathrm{U} / \mathrm{mL}$ penicillin/streptomycin. J-LAT 10.6 cells were plated in 96 well u-bottom plates at a cell density of $5 \times 10^{5} / 100 \mu \mathrm{L}$. Cells were then treated with a concentration gradient $(1 \mu \mathrm{M}, 500 \mathrm{nM}, 100 \mathrm{nM}$, $50 \mathrm{nM}, 10 \mathrm{nM}$ ) of top epigenetic modifier 'hits' for 1 hour prior to treatment with $45 \mathrm{nM}$ Ingenol3,20-dibenzoate, a suboptimal concentration in order to visualize synergy, for 24 hours. JQ-1(+) was used as a positive control for synergy as it has been previously described to synergize with PKC agonists [31]. At 24 hours cells were stained with Fixable Viability Dye eFluor ${ }^{\circledast} 450$ and fixed as described above. Latency reversal was then measured by the percentage of GFP ${ }^{+}$cells by flow cytometry as described above.

\section{Ex vivo cell culture, qPCR, and cytokine measurement}

PBMCs were isolated from HIV-1 positive aviremic donors prior to the isolation of resting CD4 ${ }^{+}$T cells (rCD4s) (EasySep ${ }^{\mathrm{TM}}$ Human Resting CD4 ${ }^{+} \mathrm{T}^{\mathrm{T}}$ Cell Isolation Kit, Cat \# 17962, StemCell Technologies). rCD4s were cultured at a density of $5 \times 10^{6} / \mathrm{mL}$ in RPMI supplemented with $10 \% \mathrm{FBS}$ and $5 \mathrm{U} / \mathrm{mL}$ penicillin/streptomycin. As Panobinostat was seen to be the most potent epigenetic modifier in reducing intracellular IL-6 and synergizing with Ingenol-3,20dibenzoate at multiple concentrations, it was added to rCD4s in the presence of medium or Ingenol-3,20-dibenzoate (100nM) for 72 hours. Medium or DMSO and Dynabead Human TActivator $\alpha C D 3 / \alpha C D 28$ ( $\alpha C D 3 / \alpha C D 28)$ (Cat\# 111.32D) stimulated cells were used as controls.

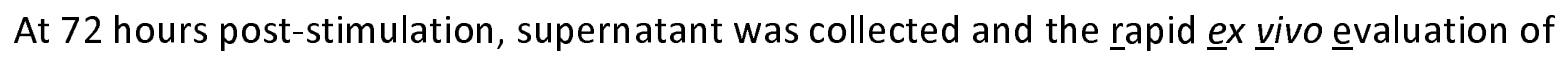
anti-latency assay (REVEAL) assay was performed as previously described [32] to quantify viral release. Statistical analysis was performed using software from GraphPad Prism Version 5.0f (GraphPad Software, Inc., San Diego CA). Statistical significance was calculated using a nonparametric Wilcoxon matched-pairs signed rank test. Additionally, supernatant was collected to measure ex vivo cytokine secretion. Supernatant was sent to ARUP Laboratories to measure ex vivo cytokine secretion via a commercially available quantitative multiplex bead assay to measure the following cytokines: interferon gamma (IFN- $\gamma$ ), tumor necrosis factor alpha (TNF$\alpha$ ), interleukin (IL) 1 beta (IL-1 $\beta$ ), IL-2, soluble IL-2 receptor (IL-2r), IL-4, IL-5, IL-6, IL-8, IL-10, IL- 
12, and IL-13. Percent change in the secretion of pro-inflammatory cytokines compared to Ingenol-3,20-dibenzoate alone treated cells was calculated with GraphPad Prism Version 5.0f (GraphPad Software, Inc., San Diego CA).

\section{Results}

As HDACi have been previously described to inhibit cytokine secretion [24-26] and to synergize with PKC agonists [27-29], we investigated the ability of HDACi and other epigenetic modifiers to reduce pro-inflammatory cytokine secretion upon latency reversal with PKC agonists. A screen of 96 epigenetic modifiers was conducted to identify epigenetic modifier 'hits' that reduced the induction of pro-inflammatory cytokines during latency reversal with Ingenol 3,20-dibenzoate. Nine epigenetic modifiers from the epigenetic library were identified as reducing intracellular IL- 6 by fourfold or greater when combined with Ingenol-3,20dibenzoate (Figure 1). These 'hits' include six HDACi (Chemical Abstracts Service (CAS) \# 743420-02-2 (Chidamide), CAS \# 183506-66-3 (Apicidin), CAS \# 209783-80-2 (Entinostat), CAS \# 38937-66-5 (Suberhydroxamic Acid (SBHA)), CAS \# 382180-17-8 (Pyroxamide), CAS \# 40495080-7 (Panobinostat)), one mixed lineage leukemia (MLL) inhibitor Cas \# 890190-22-4 (WDR50103), and two compounds categorized as miscellaneous as they did not have the same function as other epigenetic modifiers in the screen. Out of the two 'hits' categorized as miscellaneous, the compound Cas \# 160003-66-7 (Iniparib) has an unknown function and compound Cas \# 1207113-88-9 (CCG-100602) is an inhibitor of Rho pathway mediated signaling [33]. The positive control Ruxolitinib also reduced intracellular IL-6 by greater than fourfold.

Compounds identified as 'hits' for their ability to suppress cytokine production were then tested for synergy with PKC agonist Ingenol-3,20-dibenzoate in J-LAT 10.6 cells [30], as calculated by the Bliss Independence model [34]. Upon visualization of latency reversal by the percentage of GFP ${ }^{+}$cells of Ingenol-3,20-dibenzoate (control) or Ingenol-3,20-dibenzoate plus epigenetic modifier treated cells, all nine epigenetic modifiers were identified to synergize with Ingenol-3,20-dibenzoate at one or more concentrations (Figure 2). Panobinostat (10nM, 50nM, $100 \mathrm{nM}$, and 500nM) synergized with Ingenol-3-20-dibenzoate with the highest level of reactivation seen at $100 \mathrm{nM}$. Panobinostat was not tested at $1 \mu \mathrm{M}$ due high cellular toxicity. 
Apicidin treatment led to the second highest level of reactivation but was only found to be synergistic at the highest concentration tested, 1uM. Following Apicidin, compounds Entinostat

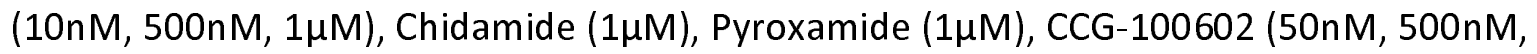
$1 \mu \mathrm{M})$, SBHA (10nM), WDR5-0103 (100nM, 500nM, 1 $\mu \mathrm{M})$, and Iniparib (100nM) were found to be synergistic with Ingenol-3,20-dibenzoate but induced low levels of reactivation. JQ-1 (+) was used as a positive control for synergy as it has been previously described to synergize with PKC agonists [31] and was found to synergize with Ingenol-3,20-dibenzoate at all concentrations except 10nM. Ruxolitinib, the positive control for intracellular IL-6 reduction, as expected, did not synergize with Ingenol-3,20-dibenzoate.

As Panobinostat was observed to be the most potent reducer of intracellular IL-6 and led to the highest level of reactivation by synergizing with Ingenol-3,20-dibenzoate at multiple concentrations, Panobinostat was then tested for the same activities ex vivo in $\mathrm{CD}^{+}{ }^{\top}$ cells isolated from HIV-1 positive aviremic patients. Panobinostat in combination with Ingenol-3,20dibenzoate was found to substantially reduce secreted levels of TNF- $\alpha$ ( $91.5 \% \pm$ standard deviation (SD) 10.4), IFN- $\gamma(98.3 \% \pm$ SD 1.0), IL-1 $\beta$ (54.8\% \pm SD 44.2), and IL-6 (83.8\% \pm SD 8.4) $(n=2)$ (donors $\mathrm{HO12}$ and H035) (Figure 3A) consistent with our results from our in vitro intracellular IL-6 screen. The combination of Ingenol-3,20-dibenzoate and Panobinostat also diminished the induction of IL-5, IL-10, IL-13, and IL-2r, with IL-12 levels remaining unchanged in both donors and IL- 8 and IL-2 levels showing inconsistent trends between donors (data not shown). However, when examining the ability of Panobinostat and Ingenol-3,20-dibenzoate to synergize ex vivo, our encouraging initial screening results in J-Lat 10.6 cells failed to reproduce in patient cells. Ingenol-3,20-dibenzoate alone and $\alpha C D 3 / \alpha C D 28$ alone significantly induced reactivation of the latent reservoir (mean $=16096 \mathrm{mRNA}$ copies $/ \mathrm{mL}, \mathrm{p}$ value $=0.0313$ and mean $=15286 \mathrm{mRNA}$ copies $/ \mathrm{mL}, \mathrm{p}$ value $=0.0078$ respectively, $\mathrm{n}=8$ ) (Figure 3B). Panobinostat alone resulted in potent viral reactivation in rCD4s from three donors (H012, H026, and H035) out of 8 (mean $=6959$ mRNA copies $/ m L, n=8$ ). The addition of Panobinostat to Ingenol-3,20dibenzoate $($ mean $=7535 \mathrm{mRNA}$ copies $/ \mathrm{mL}, \mathrm{n}=8$ ) reduced the overall response, when compared to Ingenol alone (mean $=16096 \mathrm{mRNA}$ copies $/ \mathrm{mL}, \mathrm{p}$ value $=0.0313, \mathrm{n}=8$ ). Panobinostat was determined to be antagonistic to Ingenol-3,20-dibenzoate by the Bliss 
Independence model [34]. In three out of six donors the addition of Panobinostat to Ingenol3,20-dibenzoate to rCD4s ablated reactivation completely (donors $\mathrm{H010}, \mathrm{HO2O}$, and H033) with reactivation only seen in the donors whose cells reactivated with Panobinostat alone (H012, H026, H035). A possible interpretation for these results is that Panobinostat has a potent suppressive activity over Ingenol-3,20-dibenzoate, which appears to be dominant. Reactivation in cells from two donors, H013 and H018, was only observed upon treatment with $\alpha \mathrm{CD} 3 / \alpha \mathrm{CD} 28$

\section{Discussion}

In order to eliminate the latent HIV-1 reservoir, LRAs must be evaluated based on their potency and deleterious side effects. The most potent LRAs that have emerged across multiple models of HIV-1 latency are PKC agonists [18]. However, PKC agonists are known to induce T cell activation [21] and deleterious cytokines [22, 35]. Previous reports have demonstrated that LRAs can be combined with a second pharmacological agent to suppress deleterious proinflammatory cytokines induced upon treatment with PKC agonists [22] and TCR stimulation [23]. HDACi such as SAHA [24, 26], Trichostatin A [25], and Nicotinamide [25] have been shown to reduce pro-inflammatory cytokines in the context of graft-versus-host disease [24], rheumatoid arthritis [25], and LPS-stimulated human PBMCs [26]. Additionally, HDACi have been shown to synergize with PKC agonists when reactivating latent HIV-1 [27-29]. Therefore, we sought to evaluate the ability of HDACi and other epigenetic modifiers for their ability to suppress pro-inflammatory cytokines and to synergize with the PKC agonist Ingenol-3,20dibenzoate. We found that Panobinostat, a broad-spectrum inhibitor of HDACs, reduced proinflammatory cytokines in healthy donor PBMCs and synergized with Ingenol-3,20-dibenzoate in J-LAT 10.6 cells in vitro. Furthermore, we observed that the addition of Panobinostat to Ingenol-3,20-dibenzoate substantially reduced TNF- $\alpha$, IFN- $\gamma$, IL-1 $\beta$, and IL- 6 in cells from HIV-1 positive aviremic individuals ex vivo. However, Panobinostat appeared to act in an antagonist manner to Ingenol-3,20-dibenzoate by diminishing or inhibiting reactivation in rCD4s isolated from aviremic patients. As we demonstrated a synergistic relationship between Panobinostat and Ingenol-3,20-dibenzoate in the J-LAT 10.6 in vitro HIV-1 latency model but saw an 
antagonist relationship between the two compounds in regards to latency reversal ex vivo we turned to the literature for possible explanations.

It has been well described that there are discrepancies between latently infected T cell lines and primary cell models of HIV-1 latency [18]. Spina et al. [18] demonstrated that compounds found to be strong LRAs in some cell models of latency did not induce reactivation in others which likely explains the discrepancies between our in vitro and ex vivo synergy/antagonism results. In regards to why Panobinostat would antagonize Ingenol-3,20dibenzoate induced reactivation, upon examining the literature we found several potential explanations. We found that Panobinostat, as well as another HDACi, Dacinostat, are known to induce acetylation of the chaperone protein heat shock protein 90 (Hsp90) leading to reduced binding of ATP to Hsp90 [36]. This acetylation prevents Hsp90 from interacting with its client proteins Bcr-Abl and Akt leading to reduced levels of these proteins [36, 37] and reduced levels of pAkt in chronic myeloid leukemia blast crisis (CML-BC) K562 cells and acute leukemia MV4-11 (AML) cells [38]. Bcr-Abl and Akt have both been shown to modulate NF-KB $[39,40]$ indicating that diminished Bcr-Abl and Akt protein due to acetylation of Hsp90 would likely cause reduced levels of NF-k $\beta$ expression and in turn reduced reactivation.

Additionally, Hsp90 has been shown to directly control HIV-1 reactivation from latency [41, 42], a relationship that is significantly diminished upon inhibition of Hsp90 by 17-AAG [41], an Hsp90 inhibitor. Inhibition of Hsp90 by 17-AAG was seen to significantly reduce PKC induced reactivation by Prostratin, while it did not appear to significantly reduce latency reversal by the HDACi SAHA [41]. This observation suggests that HDACi induction of latency reversal is independent of Hsp90 activity while PKC agonist activity is tied to Hsp90 and could, in theory, explain why Panobinostat alone induced reactivation in some donors but led to reduced reactivation when combined with Ingenol-3,20-dibenzoate. However, further studies are necessary before we can propose a definitive mechanism for why Panobinostat acts antagonistically towards Ingenol-3,20-dibenzoate. 


\section{Conclusions}

Panobinostat substantially decreases Ingenol-3,20-dibenzoate-induced proinflammatory cytokine release but significantly inhibits latency reversal ex vivo. While a Panobinostat and Ingenol-3,20-dibenzoate combination does not appear to be a suitable latency reversal combination therapy, other epigenetic modifiers could potentially be used in a combination therapy with PKC agonists to reduce the induction of pro-inflammatory cytokines. However, further studies are necessary to determine the effects of these epigenetic modifiers on latency reversal when used in combination with PKC agonists.

\section{Acknowledgements}

The authors express their sincere gratitude to the study participants for their continued participation in this work and ongoing translational research. This work was supported in part by the NIH National Institute of Allergy and Infectious Diseases R21 Al122377-01 (VP), NIH/University of Pennsylvania UM1AI126620 (VP), NIH National Institute of Allergy and Infectious Diseases Ruth L. Kirschstein National Research Service Award 2T32AI055434-11 (ETL), NIH National Heart, Lung and Blood Institute Grant R01 HL126547 ( AMS), the NIH National Institute of Allergy and Infectious Diseases R21 AI124823 (LM), the Doris Duke Charitable Foundation Clinical Scientist Development Award CSDA201612 (AMS, RAN). These funding organizations played no role in the design of the study, collection, analysis or interpretation of data nor the writing of this manuscript. The content of this manuscript is solely the responsibility of the authors and does not necessarily represent the official views of the NIH.

\section{Competing interests}

The authors declare no competing interests.

\section{Ethics approval}

Heathy donors and HIV-1 positive aviremic individuals on ART were recruited for phlebotomy according to Institutional Review Board (IRB) protocols 67637 (approved May 31, 2017) and 58246 (approved Jan 4, 2017) at the University of Utah. 


\section{Figure Legends}

Figure 1. Epigenetic modifiers screening identifying compounds that dampen cytokine production induced by Ingenol-3,20-dibenzoate. Screening of 96 epigenetic modifiers for compounds that reduced intracellular IL-6 induced by Ingenol-3,20-dibenzoate in healthy donor PBMCs $(n=1)$ revealed nine epigenetic modifier 'hits' that reduce intracellular IL- 6 by fourfold or greater (at or below dotted line) when compared to Ingenol treatment alone. Compounds are listed by CAS number and color coded according to categorized function. All nine 'hits' were selected for further testing.

Figure 2. Epigenetic modifiers screening identifying compounds that synergize with PKC agonist Ingenol-3,20-dibenzoate in J-LAT 10.6 cells. Screening of control Ruxolitinib and nine epigenetic modifier 'hits' identified from screening of epigenetic modifiers that reduced intracellular IL-6 cytokine production. JQ1 (+) was included as a positive control for synergy with PKC agonist Ingenol-3,20-dibenzoate. Epigenetic modifiers and controls were tested in a concentration gradient from $1 \mu \mathrm{M}$ to $10 \mathrm{nM}$ (green triangle) and viral reactivation was measured by the percentage of $\mathrm{GFP}^{+}$cells. All nine epigenetic modifier 'hits' from the previous screen were identified by Bliss Independence to synergize (asterisk) with Ingenol-3,20-dibenzoate (Ing DB) at one or more concentrations when combined with Ingenol-3,20-dibenzoate (solid bars) compared to epigenetic modifiers alone (empty bars), Media alone (dotted line), and 45nM Ingenol-3,20-dibenzoate alone (solid line). Bars are colored according to the category of compound: red (HDACi); yellow (MLL inhibitor); orange (BET inhibitor); sea green (JAK inhibitor).

Figure 3A. Panobinostat reduces pro-inflammatory cytokine release induced by Ingenol-3,20dibenzoate ex vivo. Bars represent the mean change in pro-inflammatory cytokine concentrations in the supernatant of rCD4s isolated from HIV-1 positive aviremic individuals ( $\mathrm{n}$ $=8$ ) and treated with Panobinostat and Ingenol-3,20-dibenzoate for 72 hours compared to Ingenol-3,20-dibenzoate alone. Standard deviation is indicated by error bars. 
Figure 3B. Panobinostat antagonizes Ingenol-3,20-dibenzoate induced viral reactivation ex vivo. Treatment of rCD4 cells isolated from HIV-1 positive aviremic individuals $(n=8)$ with Panobinostat resulted in viral reactivation in three donors (H012, H026, and H035) out of eight. Ingenol-3,20-dibenzoate treatment significantly increased viral latency reactivation by inducing latency reversal in six out of eight donors. The combined treatment of Panobinostat and Ingenol-3,20-dibenzoate resulted in reactivation only in donors seen to reactivate with Panobinostat alone treatment (H012, H026, and H035). The positive control $\alpha C D 3 / \alpha C D 28$ resulted in viral reactivation in all donors. Data from donors H013, H018, H020, and HO26 was previously published [32], excluding the Panobinostat and Ingenol-3,20-dibenzoate combined data. $*$ p value $<0.05 ; * * \mathrm{p}$ value $<0.01$

\section{References}

1. Gulick RM, Mellors JW, Havlir D, Eron JJ, Gonzalez C, McMahon D, Richman DD, Valentine FT, Jonas L, Meibohm A, Emini EA, Chodakewitz JA. Treatment with indinavir, zidovudine, and lamivudine in adults with human immunodeficiency virus infection and prior antiretroviral therapy. N Engl J Med. 1997;337(11):734-9.

2. Hammer SM, Squires KE, Hughes MD, Grimes JM, Demeter LM, Currier JS, Eron JJ, Jr., Feinberg JE, Balfour HH, Jr., Deyton LR, Chodakewitz JA, FischI MA. A controlled trial of two nucleoside analogues plus indinavir in persons with human immunodeficiency virus infection and CD4 cell counts of 200 per cubic millimeter or less. AIDS Clinical Trials Group 320 Study Team. N Engl J Med. 1997;337(11):725-33.

3. Perelson AS, Essunger P, Cao Y, Vesanen M, Hurley A, Saksela K, Markowitz M, Ho DD. Decay characteristics of HIV-1-infected compartments during combination therapy. Nature. 1997;387(6629):188-91.

4. Chun TW, Stuyver L, Mizell SB, Ehler LA, Mican JA, Baseler M, Lloyd AL, Nowak MA, Fauci AS. Presence of an inducible HIV-1 latent reservoir during highly active antiretroviral therapy. Proceedings of the National Academy of Sciences. 1997;94(24):13193-7.

5. Finzi D, Hermankova M, Pierson T, Carruth LM, Buck C, Chaisson RE, Quinn TC, Chadwick K, Margolick J, Brookmeyer R, Gallant J, Markowitz M, Ho DD, Richman DD, Siliciano RF. Identification of a reservoir for HIV-1 in patients on highly active antiretroviral therapy. Science (New York, NY). 1997;278(5341):1295-300.

6. Wong JK, Hezareh M, Günthard HF, Havlir DV, Ignacio CC, Spina CA, Richman DD. Recovery of replication-competent HIV despite prolonged suppression of plasma viremia. Science (New York, NY). 1997;278(5341):1291-5.

7. Torbett BE, Goodsell DS, Richman DD, editors. The Future of HIV-1 TherapeuticsResistance is Futile. Cham: Springer International Publishing; 2015. 
8. Long L, Fox M, Sanne I, Rosen S. The high cost of second-line antiretroviral therapy for HIV/AIDS in South Africa. AIDS. 2010;24(6):915-9.

9. Saberi P, Neilands TB, Vittinghoff E, Johnson MO, Chesney M, Cohn SE. Barriers to antiretroviral therapy adherence and plasma HIV RNA suppression among AIDS clinical trials group study participants. AIDS Patient Care STDS. 2015;29(3):111-6.

10. Rintamaki LS, Davis TC, Skripkauskas S, Bennett CL, Wolf MS. Social stigma concerns and HIV medication adherence. AIDS Patient Care STDS. 2006;20(5):359-68.

11. Katz IT, Ryu AE, Onuegbu AG, Psaros C, Weiser SD, Bangsberg DR, Tsai AC. Impact of HIVrelated stigma on treatment adherence: systematic review and meta-synthesis. J Int AIDS Soc. 2013;16(3 Suppl 2):18640.

12. International ASSWGoHIVC, Deeks SG, Autran B, Berkhout B, Benkirane M, Cairns S, Chomont N, Chun TW, Churchill M, Di Mascio M, Katlama C, Lafeuillade A, Landay A, Lederman M, Lewin SR, Maldarelli F, Margolis D, Markowitz M, Martinez-Picado J, Mullins JI, Mellors J, Moreno S, O'Doherty U, Palmer S, Penicaud MC, Peterlin M, Poli G, Routy JP, Rouzioux C, Silvestri G, Stevenson M, Telenti A, Van Lint C, Verdin E, Woolfrey A, Zaia J, Barre-Sinoussi F. Towards an HIV cure: a global scientific strategy. Nat Rev Immunol. 2012;12(8):607-14.

13. Elbirt D, Mahlab-Guri K, Bezalel-Rosenberg S, Gill H, Attali M, Asher I. HIV-associated neurocognitive disorders (HAND). Isr Med Assoc J. 2015;17(1):54-9.

14. Deeks SG. HIV: Shock and kill. Nature. 2012;487(7408):439-40.

15. Spivak AM, Planelles V. HIV-1 Eradication: Early Trials (and Tribulations). Trends Mol Med. 2016;22(1):10-27.

16. Burton DR, Hangartner L. Broadly Neutralizing Antibodies to HIV and Their Role in Vaccine Design. Annu Rev Immunol. 2016;34:635-59.

17. Vandergeeten $\mathrm{C}$, Fromentin $\mathrm{R}$, Chomont $\mathrm{N}$. The role of cytokines in the establishment, persistence and eradication of the HIV reservoir. Cytokine Growth Factor Rev. 2012;23(45):143-9.

18. Spina CA, Anderson J, Archin NM, Bosque A, Chan J, Famiglietti M, Greene WC, Kashuba A, Lewin SR, Margolis DM, Mau M, Ruelas D, Saleh S, Shirakawa K, Siliciano RF, Singhania A, Soto PC, Terry VH, Verdin E, Woelk C, Wooden S, Xing S, Planelles V. An in-depth comparison of latent HIV-1 reactivation in multiple cell model systems and resting CD4+ T cells from aviremic patients. PLoS Pathog. 2013;9(12):e1003834.

19. Marsden MD, Loy BA, Wu X, Ramirez CM, Schrier AJ, Murray D, Shimizu A, Ryckbosch SM, Near KE, Chun TW, Wender PA, Zack JA. In vivo activation of latent HIV with a synthetic bryostatin analog effects both latent cell "kick" and "kill" in strategy for virus eradication. PLoS Pathog. 2017;13(9):e1006575.

20. Genot EM, Parker PJ, Cantrell DA. Analysis of the role of protein kinase C-alpha, -epsilon, and -zeta in T cell activation. J Biol Chem. 1995;270(17):9833-9.

21. Korin YD, Brooks DG, Brown S, Korotzer A, Zack JA. Effects of prostratin on T-cell activation and human immunodeficiency virus latency. J Virol. 2002;76(16):8118-23.

22. Spivak AM, Larragoite ET, Coletti ML, Macedo AB, Martins L, Bosque A, Planelles V. Janus kinase inhibition suppresses PKC-induced cytokine release without affecting HIV-1 latency reversal ex vivo. Retrovirology. 2016;13(1):88. 
23. Martin AR, Pollack RA, Capoferri A, Ambinder RF, Durand CM, Siliciano RF. Rapamycinmediated mTOR inhibition uncouples HIV-1 latency reversal from cytokine-associated toxicity. J Clin Invest. 2017;127(2):651-6.

24. Reddy P, Maeda Y, Hotary K, Liu C, Reznikov LL, Dinarello CA, Ferrara JL. Histone deacetylase inhibitor suberoylanilide hydroxamic acid reduces acute graft-versus-host disease and preserves graft-versus-leukemia effect. Proc Natl Acad Sci U S A. 2004;101(11):3921-6. 25. Grabiec AM, Krausz S, de Jager W, Burakowski T, Groot D, Sanders ME, Prakken BJ, Maslinski W, Eldering E, Tak PP, Reedquist KA. Histone deacetylase inhibitors suppress inflammatory activation of rheumatoid arthritis patient synovial macrophages and tissue. J Immunol. 2010;184(5):2718-28.

26. Leoni F, Zaliani A, Bertolini G, Porro G, Pagani P, Pozzi P, Dona G, Fossati G, Sozzani S, Azam T, Bufler P, Fantuzzi G, Goncharov I, Kim SH, Pomerantz BJ, Reznikov LL, Siegmund B, Dinarello CA, Mascagni P. The antitumor histone deacetylase inhibitor suberoylanilide hydroxamic acid exhibits antiinflammatory properties via suppression of cytokines. Proc Natl Acad Sci U S A. 2002;99(5):2995-3000.

27. Laird GM, Bullen CK, Rosenbloom DI, Martin AR, Hill AL, Durand CM, Siliciano JD, Siliciano RF. Ex vivo analysis identifies effective HIV-1 latency-reversing drug combinations. J Clin Invest. 2015;125(5):1901-12.

28. Perez M, de Vinuesa AG, Sanchez-Duffhues G, Marquez N, Bellido ML, Munoz-Fernandez MA, Moreno S, Castor TP, Calzado MA, Munoz E. Bryostatin-1 synergizes with histone deacetylase inhibitors to reactivate HIV-1 from latency. Curr HIV Res. 2010;8(6):418-29.

29. Lu HK, Gray LR, Wightman F, Ellenberg P, Khoury G, Cheng WJ, Mota TM, Wesselingh S, Gorry PR, Cameron PU, Churchill MJ, Lewin SR. Ex vivo response to histone deacetylase (HDAC) inhibitors of the HIV long terminal repeat (LTR) derived from HIV-infected patients on antiretroviral therapy. PLoS One. 2014;9(11):e113341.

30. Jordan A, Bisgrove $D$, Verdin $E$. HIV reproducibly establishes a latent infection after acute infection of T cells in vitro. EMBO J. 2003;22(8):1868-77.

31. Darcis G, Kula A, Bouchat S, Fujinaga K, Corazza F, Ait-Ammar A, Delacourt N, Melard A, Kabeya K, Vanhulle C, Van Driessche B, Gatot JS, Cherrier T, Pianowski LF, Gama L, Schwartz C, Vila J, Burny A, Clumeck N, Moutschen M, De Wit S, Peterlin BM, Rouzioux C, Rohr O, Van Lint C. An In-Depth Comparison of Latency-Reversing Agent Combinations in Various In Vitro and Ex Vivo HIV-1 Latency Models Identified Bryostatin-1+JQ1 and Ingenol-B+JQ1 to Potently Reactivate Viral Gene Expression. PLoS Pathog. 2015;11(7):e1005063.

32. Spivak AM, Bosque A, Balch AH, Smyth D, Martins L, Planelles V. Ex Vivo Bioactivity and HIV-1 Latency Reversal by Ingenol Dibenzoate and Panobinostat in Resting CD4(+) T Cells from Aviremic Patients. Antimicrob Agents Chemother. 2015;59(10):5984-91.

33. Evelyn CR, Wade SM, Wang Q, Wu M, Iniguez-Lluhi JA, Merajver SD, Neubig RR. CCG1423: a small-molecule inhibitor of RhoA transcriptional signaling. Mol Cancer Ther. 2007;6(8):2249-60.

34. Bliss CI. THE TOXICITY OF POISONS APPLIED JOINTLY1. Annals of Applied Biology. 1939;26(3):585-615.

35. Kulkosky J, Culnan DM, Roman J, Dornadula G, Schnell M, Boyd MR, Pomerantz RJ. Prostratin: activation of latent HIV-1 expression suggests a potential inductive adjuvant therapy for HAART. Blood. 2001;98(10):3006-15. 
36. Nimmanapalli R, Fuino L, Bali P, Gasparetto M, Glozak M, Tao J, Moscinski L, Smith C, Wu J, Jove R, Atadja P, Bhalla K. Histone deacetylase inhibitor LAQ824 both lowers expression and promotes proteasomal degradation of Bcr-Abl and induces apoptosis of imatinib mesylatesensitive or -refractory chronic myelogenous leukemia-blast crisis cells. Cancer Res. 2003;63(16):5126-35.

37. Bali P, Pranpat M, Bradner J, Balasis M, Fiskus W, Guo F, Rocha K, Kumaraswamy S, Boyapalle S, Atadja P, Seto E, Bhalla K. Inhibition of histone deacetylase 6 acetylates and disrupts the chaperone function of heat shock protein 90: a novel basis for antileukemia activity of histone deacetylase inhibitors. J Biol Chem. 2005;280(29):26729-34.

38. George P, Bali P, Annavarapu S, Scuto A, Fiskus W, Guo F, Sigua C, Sondarva G, Moscinski L, Atadja P, Bhalla K. Combination of the histone deacetylase inhibitor LBH589 and the hsp90 inhibitor 17-AAG is highly active against human CML-BC cells and AML cells with activating mutation of FLT-3. Blood. 2005;105(4):1768-76.

39. Kane LP, Shapiro VS, Stokoe D, Weiss A. Induction of NF-kappaB by the Akt/PKB kinase. Curr Biol. 1999;9(11):601-4.

40. Reuther JY, Reuther GW, Cortez D, Pendergast AM, Baldwin AS, Jr. A requirement for NF-kappaB activation in Bcr-Abl-mediated transformation. Genes Dev. 1998;12(7):968-81. 41. Anderson I, Low JS, Weston $S$, Weinberger $M$, Zhyvoloup $A$, Labokha AA, Corazza $G$, Kitson RA, Moody CJ, Marcello A, Fassati A. Heat shock protein 90 controls HIV-1 reactivation from latency. Proc Natl Acad Sci U S A. 2014;111(15):E1528-37.

42. Vozzolo L, Loh B, Gane PJ, Tribak M, Zhou L, Anderson I, Nyakatura E, Jenner RG, Selwood D, Fassati A. Gyrase B inhibitor impairs HIV-1 replication by targeting Hsp90 and the capsid protein. J Biol Chem. 2010;285(50):39314-28. 
Figure 1

HDAC inhibitor

SIRT modifier

Methyltransferase inhibitor

BET inhibitor

Histone demethylase inhibitor

Histone acetyltransferase inhibitor
SAH hydrolase modifier

PAD inhibitor

MLL inhibitor

Antioxidant

HIF- $\alpha /$ PHD modifier

Miscellaneous

JAK inhibitor

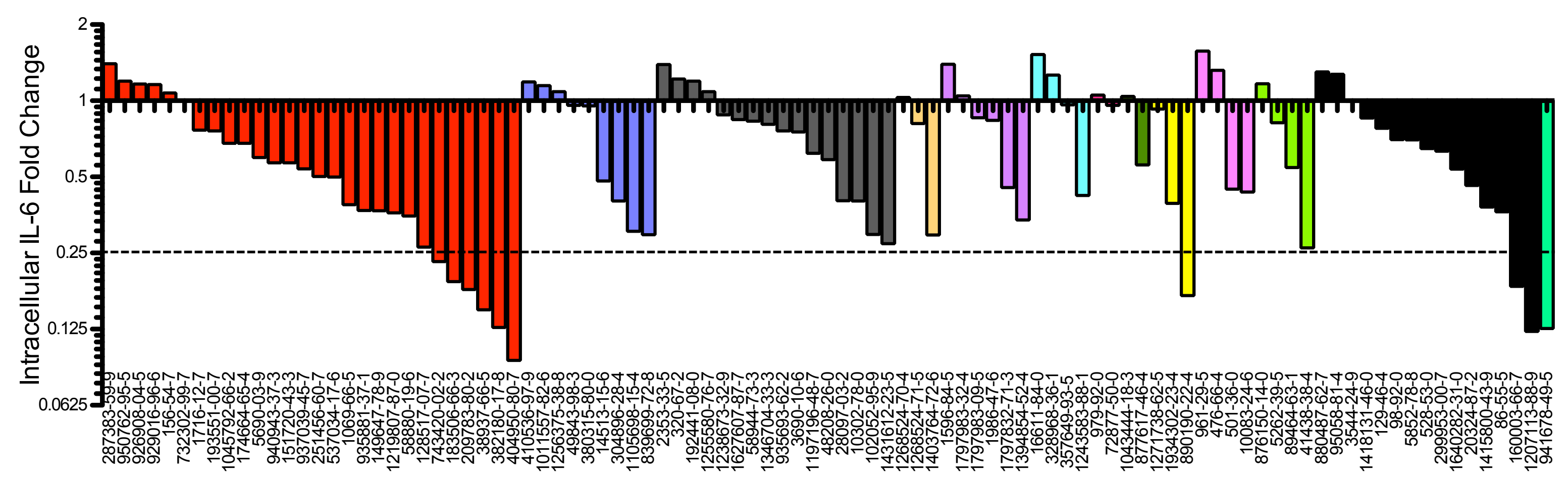




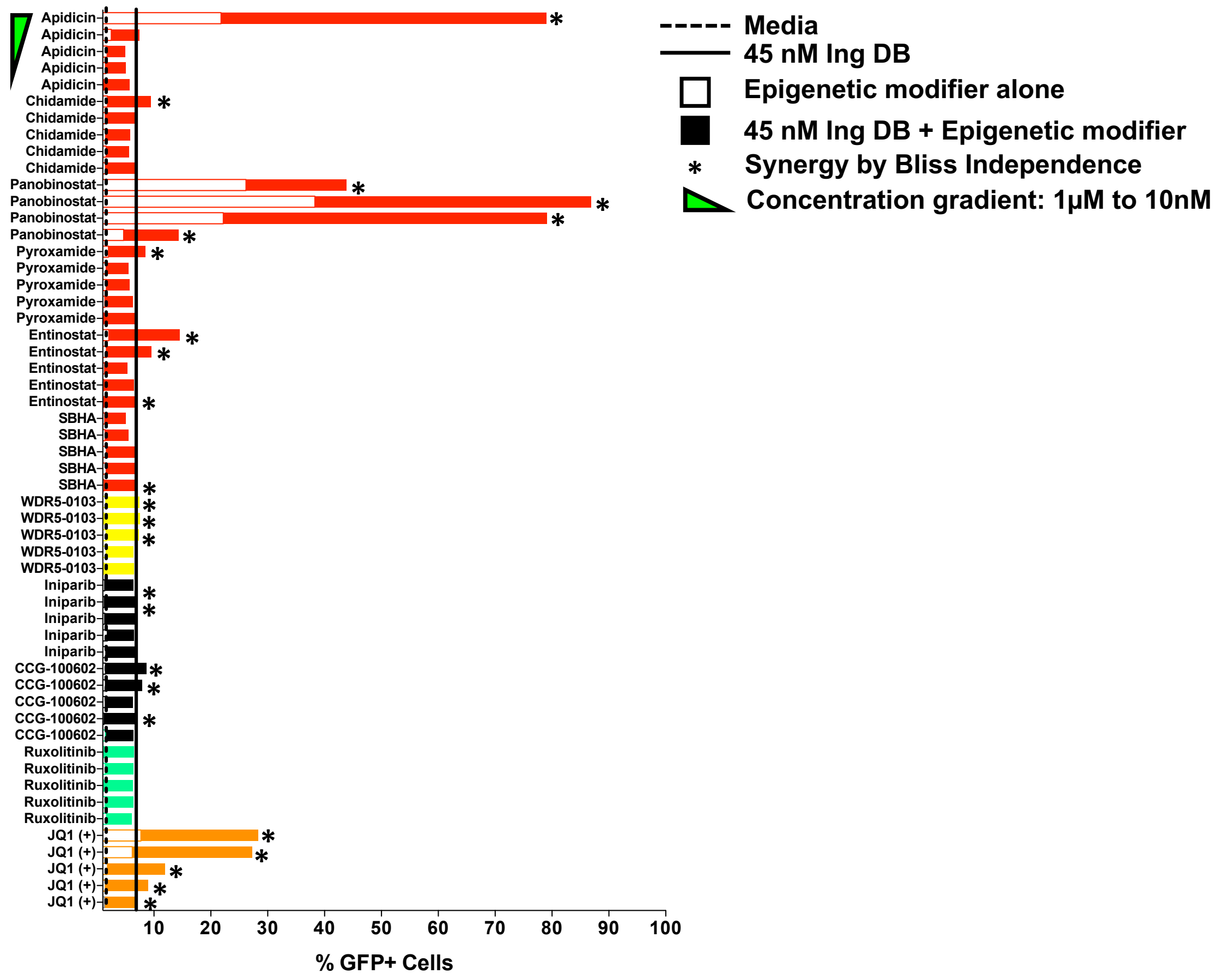




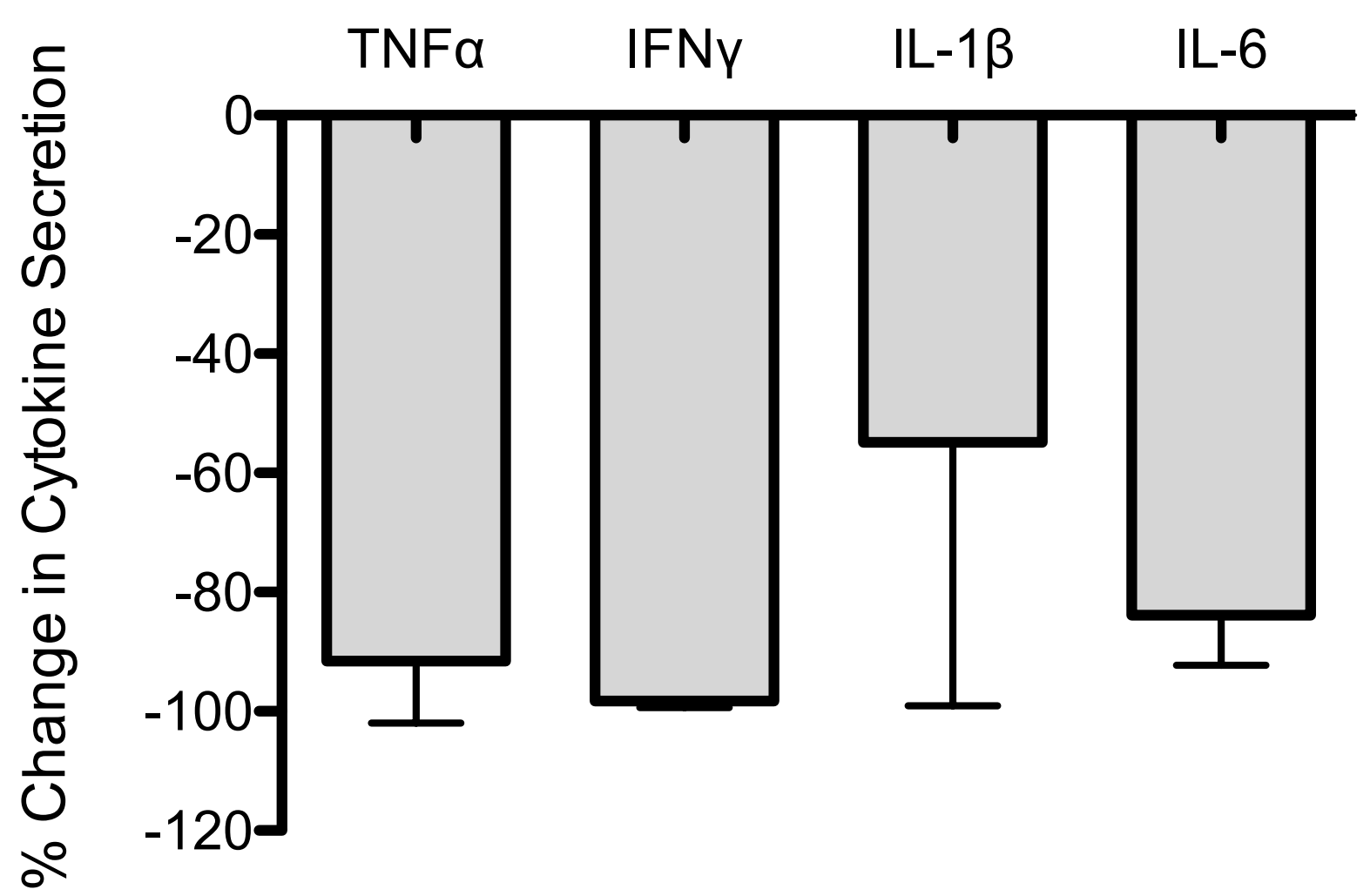


Figure 3B

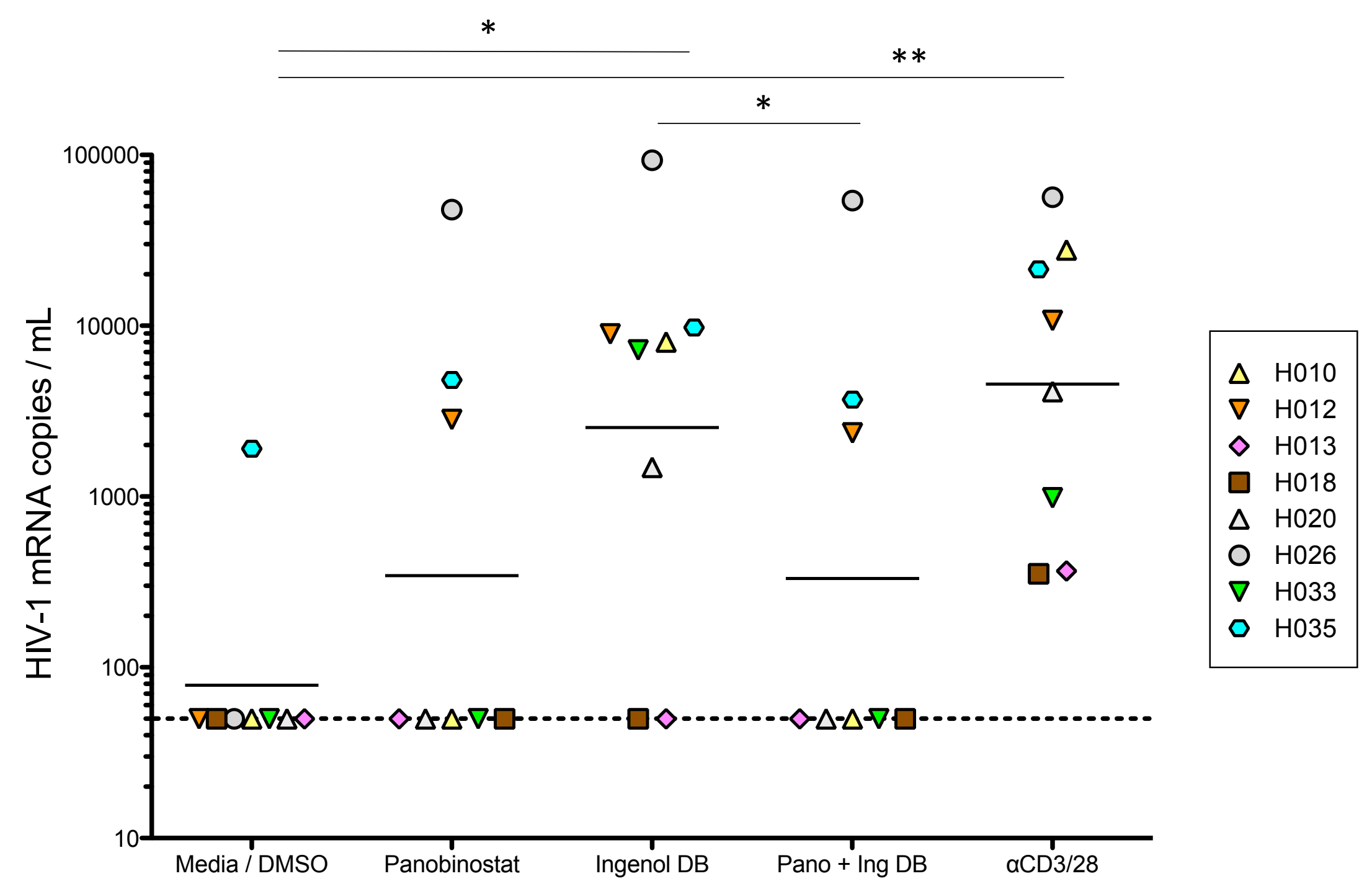

